REVISTA INTERNACIONAL DE CIENCIAS DEL DEPORTE International Journal of Sport Science

doi:10.5232/ricyde2009.01604

Rev. int. cienc. deporte

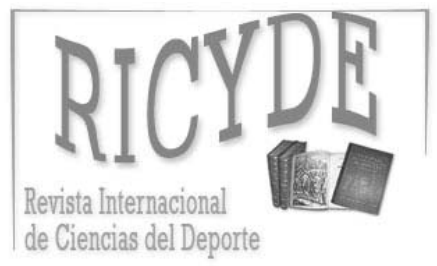

International Journal of Sport Science VOLUMEN $V$ - AÑO $V$

Páginas:52-70 ISSN:1885-3137

No 16 - Julio - 2009

\title{
Revisión sobre tipos y clasificaciones de la flexibilidad. Una nueva propuesta de clasificación. \\ Review of the Types and Classifications of Flexibility. New Proposed Classification.
}

\author{
Rafael Merino Marban \\ Emilio Fernández Rodríguez \\ Facultad de Ciencias de la Educación de la Universidad de Málaga.
}

\section{Resumen}

Al acercarse a la bibliografía para informarnos sobre conceptos y acepciones empleados al escribir sobre flexibilidad, encontramos que existe una gran maraña en torno a términos afines, conceptos empleados y tipos de flexibilidad.

Algunos autores utilizan términos distintos como sinónimos, cuando otros los diferencian claramente. El mismo término presenta una pluralidad de acepciones y conceptos distintos según quién lo utilice, dificultando sobremanera la comprensión de los distintos escritos.

En el artículo se hace un repaso por los tipos de flexibilidad y clasificaciones empleadas por los distintos autores. Poniendo en el tapete las ambigüedades, contradicciones, errores y carencias encontradas.

A partir de los datos hacemos una propuesta de cuatro clasificaciones de la flexibilidad que nos permiten abarcar y ordenar los distintos tipos de flexibilidad que aparecen en la literatura. Ofreciendo una herramienta que nos permitiría hablar con más exactitud y propiedad sobre el tema en cuestión.

Se aporta como novedad una nueva clasificación y distintas categorías en las otras clasificaciones ofreciendo un instrumento más completo a la hora de categorizar los distintos tipos de flexibilidad que son empleados por los distintos autores. Dando además un concepto claro de cada tipo de flexibilidad propuesto.

Palabras clave: flexibilidad; clasificaciones; activa, pasiva; estática; dinámica.

\begin{abstract}
Upon reviewing the literature on concepts and meanings employed when writing about flexibility, one comes across a great confusion of related terms, concepts and types of flexibility.

Some authors use different terms as synonyms, while others clearly make a distinction. The same term can have a plethora of meanings and concepts depending on who is using it, making it even more difficult to understand diverse texts.

This article reviews the types of flexibility and classifications employed by various authors, exposing the ambiguities, contradictions, errors and weaknesses encountered.

Based on this data we will propose four flexibility classifications that will permit us to include and order the various types of flexibility that appear in the literature; providing us a tool with which we can speak more precisely and with greater accuracy on the subject in question.

This article proposes a novel idea, a new classification and diverse categories within the other classifications offering a more complete instrument for categorizing the different types of flexibility used by various authors; and in addition providing a clear concept of each type of proposed flexibility.
\end{abstract}

Key words: flexibility; classifications; active; passive; static; dynamic. 


\section{Introducción}

A l acercarse a la bibliografía para informarnos sobre conceptos y acepciones - 1 empleados al escribir sobre flexibilidad, encontramos que existe una gran maraña en torno a términos afines, conceptos empleados y tipos de flexibilidad.

Algunos autores utilizan términos distintos como sinónimos, cuando otros los diferencian claramente. El mismo término presenta una pluralidad de acepciones y conceptos distintos según quién lo utilice, dificultando sobremanera la comprensión de los distintos escritos.

Se emplean vocablos provenientes de otras lenguas, que no hacen más que complicar, si cabe, el panorama nacional, más aun cuando esos términos tienen una difícil traducción (compliance, stiffness, soupless), quizás con la pretendida intención de darle más énfasis al escrito o de aclarar la situación.

En los últimos años distintos autores se han hecho eco de esta confusión terminológica y conceptual, procurando arrojar algo de luz sobre el tema. Se cita a continuación algún comentario textual al respecto:

"La amplia variedad terminológica que podamos encontrar respecto a esta cualidad física en diferentes textos especializados, provoca cierta confusión en el empleo de la misma, al utilizarse indistintamente diferentes términos otorgándoles idéntica significación (Rodríguez y Santonja, 2000)”.

"En la actualidad dentro del campo de Metodología del Entrenamiento y la Educación Física, se puede valorar, cómo no se observa una unidad de criterios en cuanto a la definición y conceptualización de las posibilidades motoras del hombre”. (Cortegaza, 2003).

Refiriéndose al termino pasivo aplicado a la flexibilidad y sus métodos, Sáez (2005) comenta: "En ningún caso, estos tipos de movilidad o posturas pueden denominarse así. Dentro del ámbito del ejercicio físico no ha lugar para la pasividad; ni siquiera en situaciones estáticas de ejercicios de relajación. Incomprensiblemente, aún siguen utilizándose dichos términos, ya obsoletos; quizás, como síntoma de que esta disciplina está muy influenciada de aspectos teóricos del campo de la medicina bastante alejados del terreno práctico”.

"El concepto de flexibilidad está íntimamente ligado a otros que por lo general se confunden y se utilizan indistintamente como sinónimos,...” (Hernández, 2007)”.

\section{Tipos de Flexibilidad y su Clasificación}

A la hora de hablar de los distintos tipos de flexibilidad y sus clasificaciones, aparece de nuevo la citada problemática.

La literatura recoge distintos tipos de flexibilidad y clasificaciones. Encontrándonos a veces algún tipo de flexibilidad que no queda recogido en estas clasificaciones. Téngase en cuenta que no todas se han elaborado siguiendo los mismos criterios.

La flexibilidad no existe como característica general del cuerpo humano sino que el grado de flexibilidad, normalmente, es muy específico tanto para cada articulación como para una acción articular concreta (Merni y col. 1981; citado en Alter, 2008; Moras, 1992; Hedrick, 2004; Bragança y col. ,2008). Platonov (2001) lo expresa claramente al indicar que la flexibilidad activa y la pasiva son específicas para cada articulación. Según 
Platonov (2001), existen deportistas que presentan un alto nivel de flexibilidad pasiva con un desarrollo escaso de la flexibilidad activa, y viceversa.

Existe en general en la comunidad científica el consenso de que la flexibilidad es específica de cada articulación y acción articular, es decir para cada movimiento y articulación. Y como los movimientos pueden ser clasificados atendiendo a variados puntos de vista, de la misma manera, las manifestaciones de la flexibilidad como expresión de las capacidades humanas de movimiento, podrán ser organizadas desde distintos enfoques.

Habría que diferenciar las causas de los distintos tipos de movimientos y en consecuencia los distintos tipos de flexibilidad, de tal manera que se encuentran distintas clasificaciones de la flexibilidad según se atienda a distintos parámetros.

Al respecto, Pareja (1995) dice lo siguiente: "un análisis de lo escrito en la literatura sobre las diferentes capacidades físicomotrices deja ver que no existen conceptos que posibiliten un criterio único de definición y de clasificación. Depende del punto o puntos de vista de quien aborda el estudio respectivo”.

\section{Clasificaciones empleadas por los distintos autores}

Antes de presentar nuestra propuesta creemos interesante hacer un recorrido por las distintos tipos de flexibilidad y clasificaciones empleados por los autores. A la hora de intentar organizar y seriar las variadas manifestaciones de la flexibilidad se ponen de manifiesto las carencias de estas taxonomías. Al encontrarnos clasificaciones que recopilan distintos tipos de flexibilidad bajo el mismo epígrafe, pero que atienden a parámetros no coincidentes.

A continuación se hace un repaso de las categorías de flexibilidad encontradas en la literatura y de los autores que las citan:

o Holland (1968; citado en Basmajian, 1982) dice que: "pueden existir dos tipos de flexibilidad: uno funcionalmente dinámico y otro que solo se mide en posiciones inactivas del cuerpo". Esta es la primera clasificación o intento de clasificación de la flexibilidad de la que tenemos constancia. Decimos intento porque el autor dice "pueden existir", en vez de existen. Si entendemos "posiciones inactivas del cuerpo" como que no hay movimiento, nos encontramos con que según Holland la flexibilidad puede ser dinámica o estática.

o Hegedüs (1984) habla de ejercicios para desarrollar la flexibilidad activa y la pasiva.

o Weineck (1988) distingue entre movilidad general y específica, y movilidad activa y pasiva. Indica que movilidad es un sinónimo de flexibilidad.

o Donskoi y Zatsiorski (1988) distinguen la flexibilidad activa y la pasiva.

o Manno (1994) y Gómez Castañeda (2004) distingue tres tipos de flexibilidad: activa, pasiva y mixta.

o Alter (1998) comenta que la flexibilidad habitualmente se clasifica en "estática o dinámica”.

o Para Esper (2000) la flexibilidad puede ser general o especial.

o Según George, Fisher y Vehrs (1999; citado en Valbuena, 2007), Gleim and McHugh (1997; citado en Vidal, 2004) y Arregui (2001) la flexibilidad puede ser estática o dinámica.

o Platonov (2001) distingue la flexibilidad activa, pasiva y también la movilidad anatómica. Según el autor, el término flexibilidad es más adecuado para valorar la 
movilidad general de las articulaciones de todo el cuerpo. Cuando se habla de una articulación en concreto, es más correcto hablar de su movilidad.

o González y col. (2001), describen tres tipos de flexibilidad: anatómica, activa y pasiva.

o Mora (1989), Vázquez y col. (1997), Platonov y Bulatova (1998), Rivera (2000), Gianikellis y col. (2003), Cadierno (2003) y Meléndez (2005) clasifican la flexibilidad en activa o pasiva.

o Según González (2005) la variedad en que se manifiesta la flexibilidad puede ser clasificada como: activa, pasiva, anatómica y cinética.

o González (2005) presenta una segunda clasificación de la flexibilidad referida al taekwondo con las siguientes categorías: activa general, activa específica, pasiva general y pasiva específica.

o Según Kim los tipos de flexibilidad (2006) son: estática, funcional y activa (pudiendo ser estática o dinámica).

o Vrijens (2006) por una parte distingue la flexibilidad general y específica y, por otra, la flexibilidad activa y pasiva.

o Según Alter (2008) algunas de las categorías más comunes de la flexibilidad son las siguientes: estática, balística, dinámica o funcional y activa, que puede ser estática o dinámica.

Conforme a los datos encontrados coincidimos con Bragança y cols (2008) en que la mayoría de los autores clasifican la flexibilidad en activa o pasiva. Aunque también destacamos el empleo de estática o dinámica. Y habría que mencionar el empleo de otras dos categorías en la literatura de manera habitual, nos referimos a la flexibilidad general y específica.

Analizando los datos fácilmente se deducen tres clasificaciones para poder ordenar las distintas categorías encontradas. Pero algunos de los tipos empleados no encuentran cabida en estas taxonomías, por lo que consideramos interesante presentar una cuarta clasificación que nos permite organizar y abarcar la variedad encontrada.

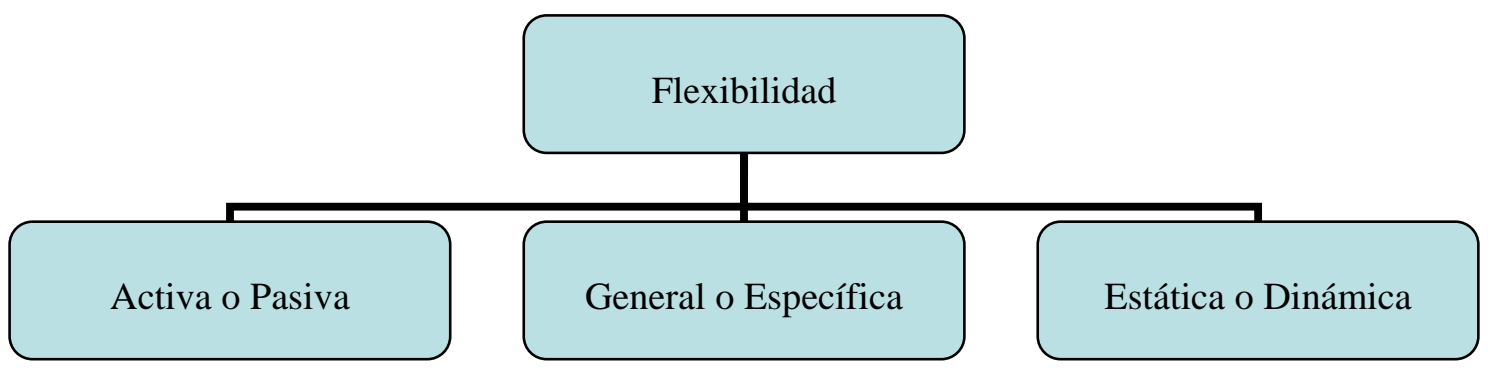

Fig.1. Clasificaciones más empleadas en la literatura.

Mención aparte merece Pareja (1995) cuando nos habla de la posibilidad de conceptuar y clasificar la flexibilidad de diversas maneras: "a partir de las necesidades de su manifestación (general, especial), de la forma de movimiento (dinámica, estática), de la participación o no de la musculatura agonista en el ejercicio de flexibilidad (activa, pasiva), del grado de expresión cuantitativa de movimiento (cantidad de elongamiento muscular y de desplazamiento angular de la articulación), entre otras conocidas”.

Este autor en su escrito manifiesta la importancia de adoptar diferentes puntos de vista en el estudio de la flexibilidad, pero no se detiene en explicar y ofrecer conceptos de las clasificaciones que cita. Solo aclara y explica un poco las clasificaciones de Fetz (dinámica o estática y pasiva o activa) y de Matdejev (absoluta, de trabajo y residual). 
A continuación mostramos una serie de tablas resumen que reúnen los tipos de flexibilidad encontrados en la literatura y los autores que los emplean. Cuando algún autor citado en otro apartado de este artículo no aparezca en estas tablas, se deberá básicamente a que emplea 2 o más términos como sinónimos del mismo tipo de flexibilidad cuando a nuestro juicio no lo son.

Tabla 1. Autores que citan la flexibilidad activa y pasiva.

\begin{tabular}{|c|c|}
\hline Activa & Pasiva \\
\hline Hegedüs (1984) & Hegedüs (1984) \\
\hline Weineck (1988) & Weineck (1988) \\
\hline Donskoi y Zatsiorski (1988) & Donskoi y Zatsiorski (1988) \\
\hline Mora (1989) & Mora (1989) \\
\hline Manno (1994) & Manno (1994) \\
\hline Pareja (1995) & Pareja (1995) \\
\hline Fetz & Fetz \\
\hline Vázquez y col. (1997) & Vázquez y col. (1997) \\
\hline Platonov y Butalova (1998) & Platonov y Butalova (1998) \\
\hline Rivera (2000) & Rivera (2000) \\
\hline González y col. (2001) & González y col. (2001) \\
\hline Platonov (2001) & Platonov (2001) \\
\hline Gianikellis y col. (2003) & Gianikellis y col. (2003) \\
\hline Cadierno (2003) & Cadierno (2003) \\
\hline Failde (2003) & Gómez (2004) \\
\hline Gómez (2004) & González (2005) \\
\hline González (2005) & Meléndez (2005) \\
\hline Meléndez (2005) & Vrijens (2006) \\
\hline Vrijens (2006) & \\
\hline $\operatorname{Kim}(2006)$ & \\
\hline Alter (2008) & \\
\hline
\end{tabular}

Tabla 2. Autores que citan la flexibilidad estática y dinámica.

\begin{tabular}{|l|l|}
\hline Estática & Dinámica \\
\hline Holland (1968) & Holland (1968) \\
\hline Gleim and McHugh (1997) & Gleim and McHugh (1997) \\
\hline Fetz & Fetz \\
\hline Pareja (1995) & Pareja (1995) \\
\hline George, Fisher y Vehrs (1999) & George, Fisher y Vehrs (1999) \\
\hline Arregui y Martínez (2001) & Arregui y Martínez (2001) \\
\hline Kim (2006) & Kim (2006) \\
\hline Alter (2008) & Alter (2008) \\
\hline
\end{tabular}

Tabla 3. Autores que citan la flexibilidad general y específica.

\begin{tabular}{|l|l|}
\hline General & Específica \\
\hline Weineck (1988) & Weineck (1988) \\
\hline Pareja (1995) & Pareja (1995) \\
\hline Esper (2000) & Esper (2000) \\
\hline Serrato (2004) & Serrato (2004) \\
\hline González (2005) & González (2005) \\
\hline Vrijens (2006) & Vrijens (2006) \\
\hline
\end{tabular}

Tabla 4. Autores que citan la flexibilidad mixta, anatómica, funcional y balística o cinética.

\begin{tabular}{|l|l|l|l|}
\hline \multicolumn{1}{|c|}{ Mixta } & \multicolumn{1}{|c|}{ Anatómica } & Funcional & Balística o cinética \\
\hline Manno (1994) & González y col. (2001) & Kim (2006) & González (2005) \\
\hline Gómez (2004) & González (2005) & & Alter (2008) \\
\hline
\end{tabular}




\section{Propuesta de clasificaciones}

Presentamos cuatro clasificaciones que permiten abarcar las variadas y complejas posibilidades de expresarse que tiene esta cualidad física.

Coincidimos plenamente con Pareja (1995) en considerar que: "se deben tener en cuenta las diferentes formas de manifestación de una capacidad física porque esto permite una mayor comprensión de los elementos teórico-prácticos inherentes a ella, tales como los pedagógicos, los fisiológicos, y los bioquímicos”.

Los cuatro enfoques desde los cuales elaboramos nuestra propuesta son los siguientes:

1. Según la aplicación de las fuerzas que intervienen en el movimiento (Flexibilidad de fuerza).

2. Según haya o no movimiento (Flexibilidad cinética).

3. Según la cantidad de articulaciones involucradas (Flexibilidad cuantitativa).

4. Según los requerimientos de movilidad (amplitud de movimiento) de la actividad a desarrollar (Flexibilidad a demanda).

Se ha puesto un nombre a cada clasificación porque de esta manera pensamos que será más fácil recordarlas al asociarlas con un término significativo con el punto de vista desde el que se elaboran. 
Merino, R.; Fernández, E. (2009). Revisión sobre tipos y clasificaciones de la flexibilidad. Una nueva propuesta de clasificación. Revista Internacional de Ciencias del Deporte. 16(5), 52-70.

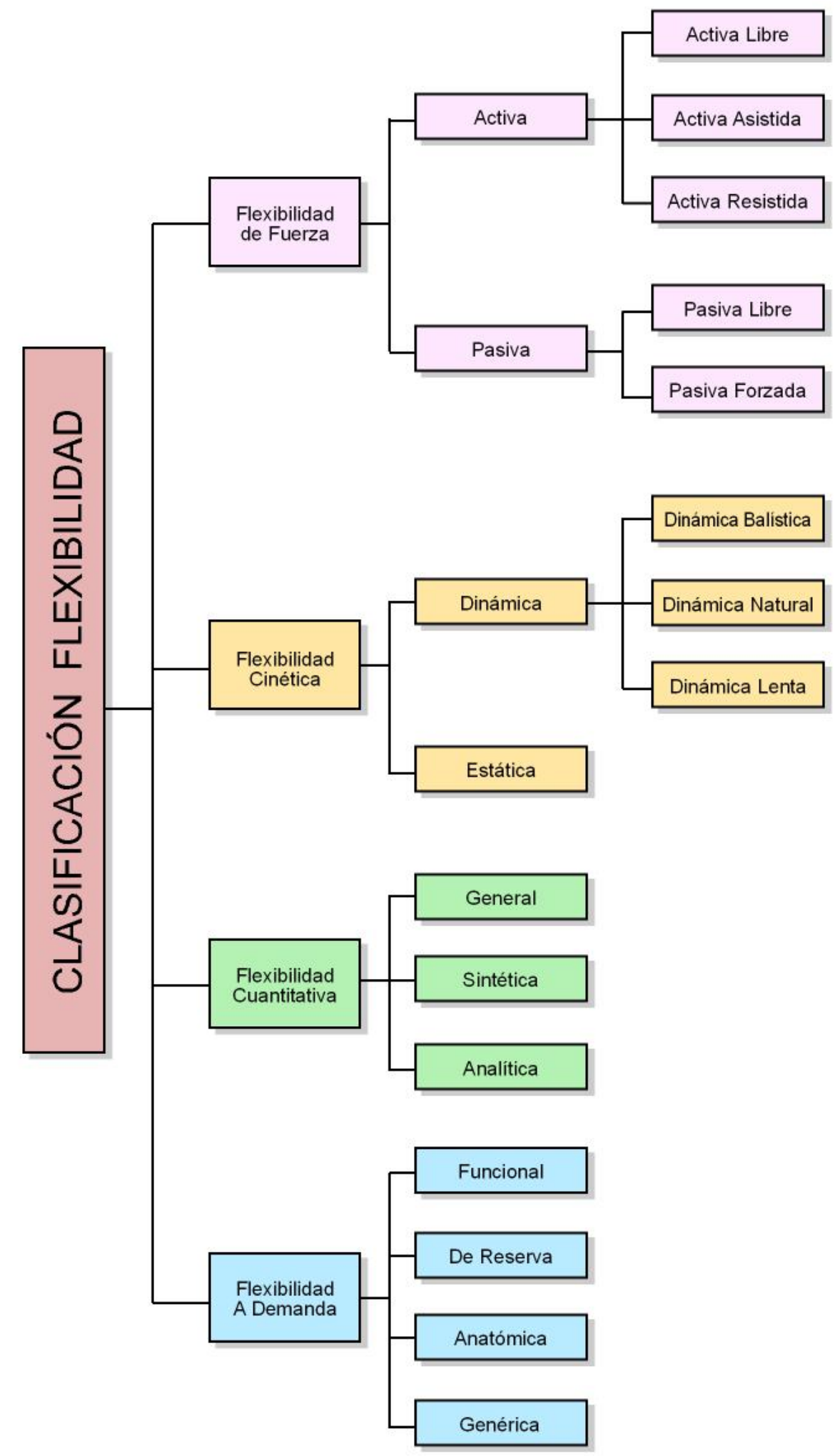

Fig. 2. Las cuatro clasificaciones de la flexibilidad. 
De esta forma a continuación presentamos las cuatro clasificaciones que, pensamos, permiten sistematizar los distintos tipos empleados en la literatura, clarificando y aportando un nuevo enfoque y organización más actual.

Se presenta cada clasificación con sus respectivas categorías y definiciones, para a continuación comentar de manera pormenorizada las ambigüedades, errores o contradicciones encontradas en la literatura.

1. Según cómo se aplican las fuerzas que intervienen en el movimiento.

Básicamente la flexibilidad se divide en activa, cuando la musculatura del sujeto se contrae para movilizar la o las articulaciones involucradas y pasiva cuando ésta musculatura no se contrae, es otra fuerza la que produce la acción. Atendiendo a este criterio nos encontramos con las siguientes categorías:

1.1. Flexibilidad activa: Capacidad para alcanzar grandes excursiones articulares gracias a la contracción de los músculos implicados (los que pertenecen a dichas articulaciones).

Ésta a su vez se puede subdividir en:

1.1.1. Flexibilidad Activa Libre: Capacidad para alcanzar grandes excursiones articulares gracias a la contracción de los músculos implicados sin que intervenga ninguna otra fuerza, (ni siquiera la fuerza de la gravedad).

1.1.2. Flexibilidad Activa Ayudada o Asistida: Capacidad para alcanzar grandes excursiones articulares gracias a la contracción de los músculos implicados y a la ayuda de otra fuerza externa.

1.1.3. Flexibilidad Activa Resistida: Capacidad para alcanzar grandes excursiones articulares gracias a la contracción de los músculos implicados mientras una fuerza externa aumenta la intensidad de la contracción.

1.2. Flexibilidad pasiva: Capacidad para alcanzar grandes excursiones articulares bajo la acción de fuerzas externas, sin que se contraiga la musculatura de la o las articulaciones movilizadas.

Esta a su vez se puede subdividir en:

1.2.1. Flexibilidad Pasiva Relajada: Capacidad para alcanzar grandes excursiones articulares bajo la acción de una única fuerza externa: el peso del cuerpo y, sin que se contraiga la musculatura de la o las articulaciones movilizadas.

1.2.2. Flexibilidad Pasiva Forzada: Capacidad para alcanzar grandes excursiones articulares bajo la acción de fuerzas externas. Además del peso corporal, actúa, al menos, otra fuerza externa (compañero, máquina), sin que se contraiga la musculatura de la o las articulaciones movilizadas.

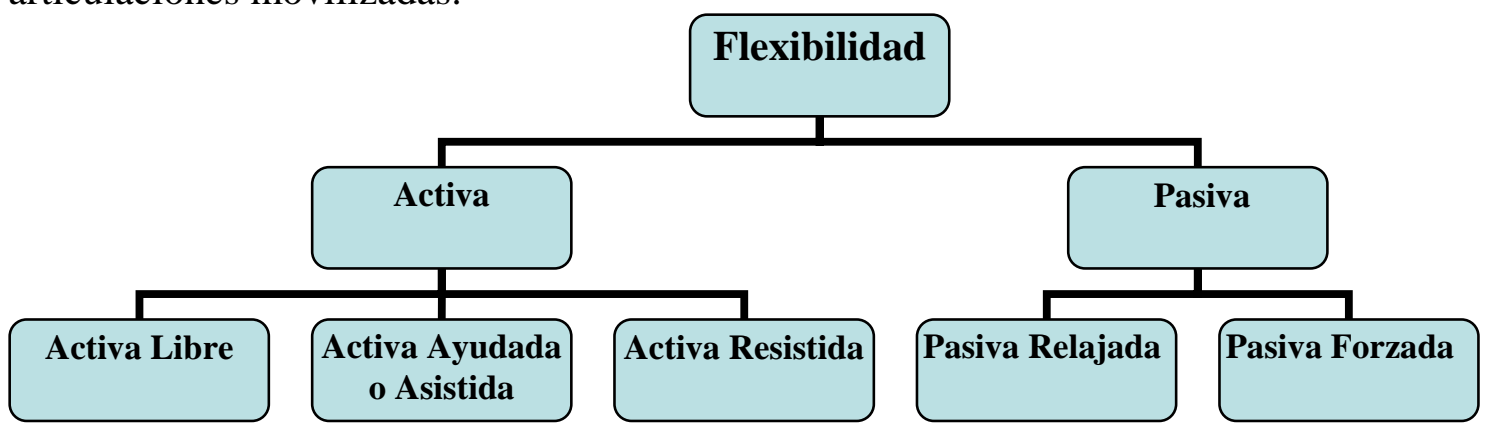

Fig. 3. Tipos de flexibilidad según como se aplican las fuerzas que intervienen. 


\section{Observaciones sobre la flexibilidad activa}

$\checkmark$ Al hablar de la flexibilidad activa casi todos los autores coinciden en que los movimientos son realizados por la musculatura implicada de la o las articulaciones movilizadas, sin necesidad de ayuda externa. Esto implica que no se plantean la existencia de la activa asistida ni de la activa resistida.

$\checkmark$ Failde (2003) y Alter (2008) dicen que: “la flexibilidad activa se refiere al rango de movilidad realizado a través de la utilización voluntaria de los músculos propios sin oponer resistencia". Con lo cual la activa resistida no podría existir.

$\checkmark$ Vázquez y col. (1997), González y col. (2001), Cadierno (2003), González (2005) y Kim (2006) hacen hincapié en que el movimiento debe realizarse sin ayuda externa de ningún tipo, usando solo los músculos de la persona.

$\checkmark$ González (2005) es el único que especifica que dentro de estas fuerzas externas se debe incluir también al propio peso corporal, que tampoco puede ayudar o facilitar el movimiento. Si tenemos en cuenta que en muchos movimientos, según como se coloque el cuerpo, el propio peso del segmento corporal o del propio cuerpo puede disminuir o aumentar la amplitud de movimiento. Deberíamos considerar el propio peso del segmento implicado como una fuerza externa a tener en cuenta.

$\checkmark$ Kim (2006) comenta sobre la flexibilidad activa que es un término un tanto confuso porque ésta puede ser estática o dinámica. No tiene por qué haber confusión ya que hablamos de clasificaciones diferentes (una referida a la actividad muscular y otra al punto de vista de que exista movimiento o no). Para este autor solo existiría la activa libre.

$\checkmark$ Fleischman (citado en Colado, 1996), Bagur y Ayuso (2001) y Serrato (2004) hablan de flexibilidad activa o dinámica como sinónimos, cuando sin embargo el primer término hace referencia a que hay contracción muscular y el segundo a que hay movimiento. Serían términos aplicables a dos categorías de flexibilidad pertenecientes a distintas clasificaciones. Es decir la flexibilidad activa a su vez puede ser dinámica o estática. Al igual que la dinámica podría ser activa o pasiva. Pero no pueden emplearse como términos sinónimos.

$\checkmark$ Donskoi y Zatsiorski (1988), Vázquez y otros (1997), Platonov (2001) y González Núñez (2005) se refieren a ella como a una capacidad, mientras Vrijens (2007) nos habla de la agilidad en el movimiento.

$\checkmark$ Weineck (1988), Harre (citado en Colado, 1996), González y col. (2001), Gianikellis y col. (2003) y Vrijens (2007) hablan de que el movimiento gracias a la contracción muscular debe ser máximo. Otros en cambio no dicen que tenga que ser máxima la amplitud.

$\checkmark$ González y col. (2001) y Kim (2006) incluyen en su concepto que el movimiento es realizado por un atleta o deportista. Consideramos restrictivo el añadirlo, ya que cualquier sujeto independientemente de ser o no deportista puede hacer uso de la flexibilidad activa.

$\checkmark$ Donskoi y Zatsiorski (1988), Weineck (1988), Harre (citado en Colado, 1996), Vázquez y col. (1997), Platonov (2001) y Gianikellis y col. (2003) indican que el movimiento es en una articulación dada. Estarían entonces hablando de flexibilidad activa específica. Pero, ¿qué pasa si se movilizan varias articulaciones?, entonces haríamos referencia a la flexibilidad activa sintética o general. Dependiendo del número de articulaciones involucradas en la acción. 


\section{Observaciones sobre la flexibilidad activa resistida}

$\checkmark$ La fuerza externa que obliga a la musculatura a contraerse puede tener dos sentidos de acción, provocando dos tipos de contracción muscular. Por un lado lo más común es que la musculatura realice una contracción muscular concéntrica debido a que la fuerza externa se opone al movimiento. O también puede ocurrir que la contracción sea excéntrica si la fuerza externa va en el sentido del movimiento como ocurre con los estiramientos resistidos de Cooley (2007), siendo la propia musculatura del sujeto la que vaya "frenando" a esa fuerza externa.

\section{Observaciones sobre la flexibilidad pasiva}

$\checkmark$ Fleischman (citado en Colado, 1996; y García y cols., 1996), Bagur y Ayuso (2001), Serrato (2004) y Harichaux y Medelli (2006) hablan de flexibilidad pasiva o estática, considerando ambos términos como sinónimos. A nuestro parecer no pueden utilizarse indistintamente ya que son tipos de flexibilidad pertenecientes a clasificaciones realizadas desde distintos puntos de vista. La flexibilidad pasiva indica que no hay contracción muscular y la estática que no hay movimiento. Estos autores indican que el movimiento se realiza de forma lenta.

$\checkmark$ Harre (citado en Colado, 1996), Vázquez y col. (1997) y González y col. (2001) indican que el movimiento es en una articulación dada. Con lo cual entonces estaríamos hablando de flexibilidad pasiva analítica, pero la flexibilidad pasiva puede manifestarse en dos o más articulaciones. Es decir, también podría ser general o sintética.

$\checkmark$ Para Harre (1976; citado en Weineck, 1988) y Gianikellis y col. (2003) el movimiento es realizado por un deportista. Luego cierran la posibilidad a que la flexibilidad pasiva pueda expresarse en cualquier tipo de persona.

$\checkmark$ Harre (1976; citado en Weineck, 1988), Vázquez y col. (1997), González y col. (2001), Gianikellis y col. (2003) y González (2005) hablan de la máxima amplitud de movimiento articular. Pensamos que no es necesario llegar a la máxima amplitud de movimiento articular para hacer uso de la flexibilidad pasiva o alguna de sus dos subcategorías.

\section{Según haya o no movimiento.}

El criterio seguido en esta clasificación es la existencia o no de movimiento al expresarse la flexibilidad, estableciendo dos categorías muy bien definidas. Al poder realizar este movimiento a distintas velocidades surgen de manera lógica otras tres subcategorías de la flexibilidad dinámica.

2.1. Flexibilidad dinámica: Capacidad de utilizar una gran amplitud articular durante un movimiento o una secuencia de movimientos.

Esta a su vez se puede subdividir en:

2.1.1. Flexibilidad dinámica balística: Capacidad de utilizar una gran amplitud articular durante un movimiento o una secuencia de movimientos realizados gracias al impulso e inercia posterior de un movimiento enérgico.

2.1.2. Flexibilidad dinámica natural: Capacidad de utilizar una gran amplitud articular durante un movimiento o una secuencia de movimientos realizados tanto a velocidad normal como elevada. 
2.1.3. Flexibilidad dinámica lenta: Capacidad de utilizar una gran amplitud articular durante un movimiento o una secuencia de movimientos realizados a una velocidad por debajo de lo normal.

2.2.Flexibilidad estática: Capacidad para mantener una postura en la que se emplee una gran amplitud articular.

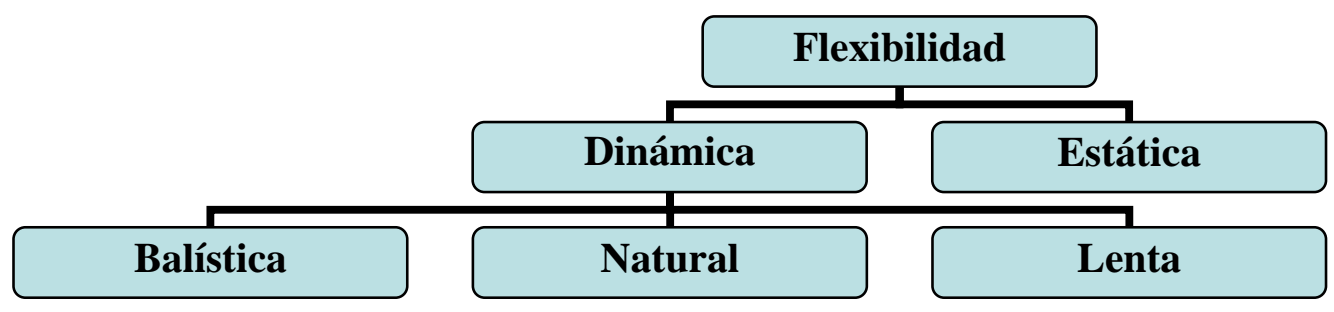

Fig. 4. Tipos de Flexibilidad según haya o no movimiento.

\section{Observaciones sobre la flexibilidad dinámica}

$\checkmark$ Consideramos apropiado el establecer estas tres subcategorías debido a los comentarios que realizan los distintos autores tomando la velocidad del movimiento como criterio para definir el tipo de flexibilidad.

$\checkmark$ Fleishman (citado en García y col., 1996), García y Pacheco (2005) y Harichaux y Medelli (2006) en su definición hacen referencia a que la flexibilidad involucra a una sola articulación. Cuando como hemos comentado anteriormente también puede involucrar a dos o más articulaciones.

$\checkmark$ Fleishman (citado en García y col., 1996), Bagur y Ayuso (2001) y García y Pacheco (2005) indican que la amplitud se alcanza gracias a una contracción muscular voluntaria del propio sujeto. Luego no podría existir la dinámica pasiva, es decir, aquella manifestación de la flexibilidad en que una fuerza externa realiza la acción. Y tampoco podría manifestarse si un aparato produjese de forma no voluntaria la contracción muscular.

$\checkmark$ Fleischman (citado en Colado, 1996), Bagur y Ayuso (2001) y Serrato (2004) hablan de flexibilidad dinámica o activa como sinónimos. Cuando no tiene por qué ser así, ya que dinámica implica que existe movimiento y activa que se produce una contracción muscular del sujeto en las articulaciones involucradas. Puede darse el caso de estar realizando movimientos de gran amplitud sin la intervención muscular del sujeto, gracias a la fuerza ejercida por un compañero. Estaríamos hablando de flexibilidad dinámica pasiva, por lo que flexibilidad dinámica no es sinónimo de activa.

$\checkmark$ Failde (2003) y Alter (2008) hablan de dinámica o funcional como sinónimos cuando se aplica a los rendimientos de una actividad física concreta. La flexibilidad dinámica no tiene por qué referirse a una actividad deportiva. Puede necesitarse para cualquier actividad de la vida cotidiana.

$\checkmark$ Dick (1993) también la denomina: cinética, balística o de rebote.

$\checkmark$ Fetz (citado por Milton; citado por Pareja, 1995) entiende por flexibilidad dinámica la ejercitación de ella con movimientos repetitivos de impulsos, insistencias y rebotes.

$\checkmark$ Failde (2003) y Alter (2008) encuentran que la flexibilidad balística se asocia comúnmente a sacudidas o rebotes llevados a través de movimientos rítmicos.

$\checkmark$ Dick (1993) y González (2005) comentan de la flexibilidad cinética, que es el movimiento que se efectúa debido al impulso de una y otra de las palancas. 
$\checkmark$ Fleishman (citado en García y col., 1996), Corbin y Noble (1980; citados en Alter, 1998), Failde (2003), García y Pacheco (2005) y Alter (2008) al hablar de flexibilidad dinámica especifican que el movimiento se realiza a velocidad normal o acelerada. Dando a entender que si se realiza lentamente el movimiento se estaría hablando de otro tipo de flexibilidad. Posiblemente flexibilidad estática, cuando para nosotros la flexibilidad estática implica que no hay movimiento.

$\checkmark$ Para Heyward (1996) la flexibilidad dinámica es una medida de la torsión o resistencia al movimiento. De manera similar para Harichaux y Medelli (2006) es la resistencia que opone una articulación a un movimiento. Y para Valbuena (2007) es la capacidad de realizar movimientos de gran amplitud en oposición a una resistencia.

$\checkmark$ Según Gleim y McHugh (citado en Vidal, 2004) se refiere a la facilidad de movimiento dentro del posible rango de movilidad.

Observaciones sobre la flexibilidad estática

$\checkmark$ Fleischman (citado en Colado, 1996 y García y col., 1996), Bagur y Ayuso (2001), Serrato (2004) y Harichaux y Medelli (2006) hablan de flexibilidad estática o pasiva como sinónimos. Como se comentó anteriormente estos términos hacen referencia a tipos de flexibilidad pertenecientes a clasificaciones de la flexibilidad realizadas desde distintos enfoques.

$\checkmark$ Para Fetz (citado por Milton; citado por Pareja, 1995), Kim (2006) y Valbuena (2007) la flexibilidad estática no implica movimiento. Son los únicos autores encontrados que expresan claramente y comparten nuestro concepto de no movimiento en este tipo de flexibilidad.

$\checkmark$ En el concepto de flexibilidad estática Fleischman (citado en Colado, 1996), Alter (1998, 2008), Bagur y Ayuso (2001) y Failde (2003) hablan de movimiento lento o de no hacer énfasis en la velocidad del movimiento. Cuando para nosotros estático significa sin movimiento.

$\checkmark$ Fleischman (citado en Colado, 1996), Bagur y Ayuso (2001) y García y Pacheco (2005) dicen que es producida por una fuerza externa. Para nosotros flexibilidad estática significa sin movimiento, y la fuerza puede ser externa, interna o ambas a la vez.

$\checkmark$ Para Heyward (1996) la flexibilidad estática es una medida del ROM (rango de movilidad) total en la articulación.

$\checkmark$ Según Gleim y McHugh (citado en Vidal, 2004) se define como el rango de movimiento disponible por una o varias articulaciones.

$\checkmark$ Manno (1994) y Gómez (2004) hablan también de la “flexibilidad mixta”, debida a la interacción de la activa y pasiva de forma variada.

No es lo mismo estático que pasivo, aunque muchos autores utilicen ambos términos indistintamente. Habría que diferenciar las causas de los distintos tipos de movimientos y en consecuencia los distintos tipos de flexibilidad. De tal manera que nos encontramos con distintas clasificaciones de la flexibilidad según se atienda a distintos parámetros.

Si cruzamos las dos clasificaciones de la flexibilidad, la que atiende a la existencia o no de movimiento y la que se categoriza según haya o no contracción muscular, se podría encontrar que la flexibilidad estática puede ser activa o pasiva. Por lo que a continuación exponemos los conceptos de estas dos posibles alternativas de la flexibilidad estática: 
o Flexibilidad estática activa: capacidad para mantener una postura en la que se emplee una gran amplitud articular gracias a la contracción isométrica de los grupos musculares implicados.

o Flexibilidad estática pasiva: capacidad para mantener una postura en la que se emplee una gran amplitud articular gracias a la ayuda externa, sin participación muscular del sujeto en las articulaciones involucradas.

Esta misma combinación de tipos de flexibilidad aparece en Valbuena (2007) al citar que: "según Alter (1986) cuando se mantienen posiciones extendidas tensionando únicamente los músculos agonistas y sinergistas, mientras los antagonistas se encuentran estirados, estamos en presencia de la flexibilidad estática activa”.

3. Según la cantidad de articulaciones involucradas.

3.1. Flexibilidad general: Capacidad de alcanzar grandes amplitudes articulares solicitando simultáneamente a muchas o a casi todas las articulaciones del cuerpo.

3.2. Flexibilidad analítica: Capacidad de alcanzar una gran amplitud articular en una sola articulación.

3.3. Flexibilidad sintética: Capacidad de alcanzar grandes amplitudes articulares en dos o varias articulaciones simultáneamente.

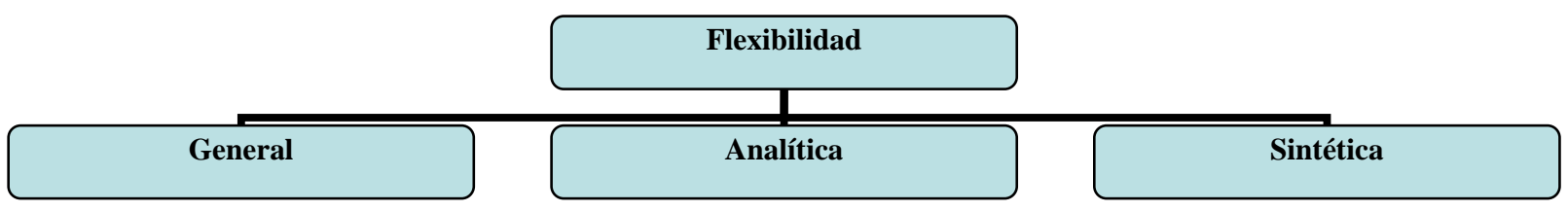

Fig. 5. Tipos de flexibilidad según el número de articulaciones involucradas.

Observaciones sobre la flexibilidad general

$\checkmark$ Weineck (1988), Colado (1996), Gisbert (citado en García y col., 1996), Serrato (2004) y Vrijens (2006) al citar esta categoría incluyen en sus conceptos la necesidad de tener en cuenta a las principales articulaciones del cuerpo (escápulohumeral, coxo-femoral y columna vertebral). En realidad no haría falta citar en el concepto a las principales articulaciones del cuerpo por considerar que al decir que intervienen muchas o casi todas las articulaciones del mismo, se sobreentiende que éstas intervienen en mayor o menor medida.

$\checkmark$ Benavent y col. (2008) emplean adecuadamente los términos y conceptos al hablarnos en su estudio de la flexibilidad general activa.

Observaciones sobre la flexibilidad analítica

$\checkmark$ Gisbert (citado en García y col., 1996) y Vrijens (2006) la relacionan con una técnica deportiva específica. Nosotros pensamos que no tiene por qué estar relacionada con una técnica deportiva concreta, pudiendo estar relacionada con cualquier actividad cotidiana.

$\checkmark$ Todos los autores encontrados, que dan un concepto de este tipo de flexibilidad, hablan de una sola articulación salvo Gisbert (citado en García Manso y col., 1996), que incluye la movilidad de una o varias articulaciones en su concepto.

Weineck (1988), Gisbert (citado en García y col., 1996), Colado (1996), Serrato (2004) y Vrijens (2006) emplean el termino de flexibilidad específica. Pero pensamos 
que cambiar la denominación de flexibilidad específica por el de analítica evita equívocos y se ajusta mejor al concepto que se pretende transmitir.

Observaciones sobre la flexibilidad sintética

$\checkmark$ Categoría que no cita ningún autor, pero que consideramos interesante incluir para completar la clasificación. Debido a que entre utilizar una, varias o casi todas las articulaciones del cuerpo existe un rango de cantidad de articulaciones muy amplio. Quedando un hueco por cubrir que la flexibilidad sintética cubre perfectamente.

4. Según los requerimientos de movilidad (amplitud de movimiento) de la actividad a desarrollar.

4.1. Flexibilidad funcional: Capacidad de alcanzar grandes amplitudes articulares necesarias para realizar una actividad específica.

4.2. Flexibilidad de reserva: Capacidad de alcanzar una amplitud articular superior a la requerida por una actividad específica para evitar rigideces que puedan afectar la coordinación del movimiento o a su nivel de expresividad.

4.3. Flexibilidad anatómica: Capacidad de alcanzar la máxima amplitud que poseen las articulaciones.

4.4. Flexibilidad genérica: Capacidad de alcanzar grandes amplitudes articulares que no sean específicas de una actividad concreta.

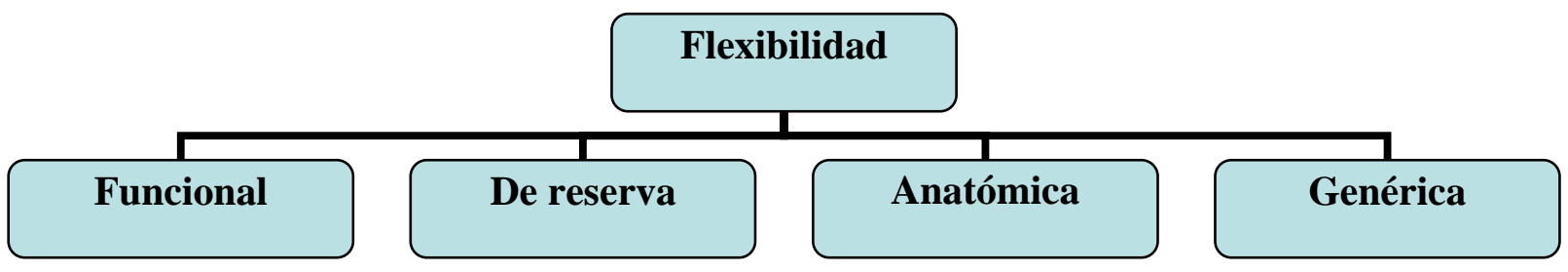

Fig. 6. Tipos de flexibilidad según los requisitos de movilidad de la actividad.

\section{Observaciones sobre la flexibilidad funcional}

$\checkmark$ Metveiev (citado en García y col., 1996) y Serrato (2004) la denominan movilidad de trabajo y Esper (2000) la llama flexibilidad especial.

$\checkmark$ Al hablar de ella, todos los autores hacen referencia a las exigencias del deporte practicado, aunque Kim (2006) y Diéguez (2007) aclaran que puede emplearse para referirse a gestos de naturaleza no deportiva. Esta última aclaración también la compartimos nosotros.

$\checkmark$ Brooks (2001) y Diéguez (2007) incluyen en su concepto que la flexibilidad funcional implica una amplitud de movimiento segura para la integridad y estabilidad articular.

\section{Observaciones sobre la flexibilidad de reserva}

$\checkmark$ Donskoi y Zatsiorski (1988) y Platonov (2001) hablan de reserva de flexibilidad para referirse a la magnitud de más que sobrepasa la amplitud máxima con que se ejecuta el movimiento.

$\checkmark$ Metveiev (citado en García y col., 1996) y Serrato (2004) la denominan residual.

$\checkmark$ González y col. (2001) y Gianikellis y col. (2003) hablan de reserva de flexibilidad para referirse a la diferencia de amplitud que existe entre la flexibilidad pasiva y 
activa. Frey (citado en Colado, 1996) denomina a esta diferencia reserva motriz. Donskoi y Zatsiorski (1988) emplean los términos déficit de la flexibilidad activa.

$\checkmark$ Atendiendo a los conceptos que nos dan los autores debemos diferenciar claramente flexibilidad de reserva de reserva de flexibilidad. La primera se refiere a un tipo de flexibilidad, que nos permite expresar esa capacidad de alcanzar una mayor amplitud para evitar rigideces. Y la segunda indica la diferencia de magnitud entre la flexibilidad pasiva y activa.

$\checkmark$ Serrato (2004) nos habla de la capacidad de movimiento del "deportista", restringiendo por lo tanto el concepto a esta población.

\section{Observaciones sobre la flexibilidad anatómica}

$\checkmark$ Platonov (2001) también la llama máxima y Metveiev (citado en García y col., 1996) absoluta o máxima.

$\checkmark$ Según Metveiev (citado en García y col., 1996) suele alcanzarse en los movimientos pasivos y forzados de cada una de las articulaciones.

$\checkmark$ Para Metveiev (citado en García y otros, 1996), González y col. (2001) y González (2005) la flexibilidad anatómica depende de la capacidad de elongación de los elementos blandos. Pero además para Platonov y Bulatova (1998), Platonov (2001) y González y col. (2001) también depende de la estructura de las articulaciones involucradas. Nosotros pensamos que en este tipo de flexibilidad la estructura de la articulación es de suma importancia, ya que la forma de la articulación condiciona sobremanera el rango de movimiento.

$\checkmark$ Platonov y Bulatova (1998) comentan que las particularidades de la estructura de cada tipo de articulación es responsable de su movilidad. "La amplitud de los movimientos depende sobre todo del grado de correspondencia entre la magnitud y la capacidad de desplazamiento de las superficies articulares. Cuanto mayor es la diferencia de la superficie (incongruencia de las articulaciones), tanto mayor será la posibilidad de desplazamiento de los huesos entre sí y mayor será el ángulo de desviación”.

Observaciones sobre la flexibilidad genérica

$\checkmark$ Colado (1996) emplea el término genérica al referirse a la flexibilidad general. Sin embargo, pensamos que es un término más adecuado para referirse a movimientos de gran amplitud que no sean específicos de una actividad concreta. Categoría que creemos necesaria y la denominación coincidiría con el término empleado por Colado.

\section{Conclusiones finales}

Desde todos los puntos de vista, se podrían cruzar las distintas clasificaciones con el objeto de precisar el tipo de flexibilidad empleado en una acción determinada. De tal manera que nos podríamos encontrar, por ejemplo, con "la flexibilidad activa dinámica asistida anatómica analítica”. Al realizar un movimiento en el que se mueve un segmento alrededor de una sola articulación en su máxima amplitud, empleando la contracción de la musculatura que atraviesa esa articulación y siendo ayudado en la ejecución por una fuerza externa al sujeto.

Por lo tanto se deben emplear las clasificaciones para ayudarnos en la tarea de analizar y estudiar esta cualidad dentro de la actividad física. Pensamos que es importante para el 
buen entendimiento el aclarar en la medida de lo posible toda esta confusión que a veces rodea a nuestra área de conocimiento.

Debido a todo este panorama terminológico y conceptual pensamos que había que aclarar todo esta confusión, por lo que resumimos a continuación nuestras aportaciones:

En la primera clasificación que diferencia entre activa y pasiva no hemos encontrado ningún autor que emplee subcategorías. Nosotros hemos añadido tres a la flexibilidad activa (activa libre, activa asistida y activa resistida) y dos a la pasiva (pasiva relajada y pasiva forzada). De esta manera pensamos ponemos nombre a capacidades existentes y facilitamos la labor de aquellos profesionales que hacen uso habitualmente de ellas.

En la segunda clasificación, según haya o no movimiento, solo encontramos dos autores que expresan claramente que la flexibilidad estática es sin movimiento. Los demás hablan de movimiento lento, lo cual para nosotros no es apropiado, ya que estático implica sin movimiento. Hemos añadido tres subcategorías a la flexibilidad dinámica ya que muchos autores hablan de distintas velocidades para referirse a este tipo de flexibilidad.

La tercera clasificación, que atiende al número de articulaciones involucradas, no existe claramente en los textos, pero se puede inferir de ellos. Aunque pensamos que se mezclan otros conceptos, al relacionar muchos autores la flexibilidad especifica con las necesidades de una actividad física concreta. Asimismo al referirnos a número de articulaciones, pensamos que el término analítica es más adecuado que el de específica. Además de todo esto añadimos la categoría de flexibilidad sintética para completar la laguna que queda entre flexibilidad general y analítica.

Y por último, la cuarta clasificación pensamos que era necesaria al abarcar categorías no recogidas en ninguna clasificación, pero empleadas algunas de ellas por ciertos autores. Añadimos la flexibilidad genérica por considerar que al existir la flexibilidad funcional, relacionada con una actividad específica, por contraposición debía existir otra categoría que no tuviese relación con actividad concreta.

\section{Referencias bibliográficas}

Alter, M. (1998). Los estiramientos. Desarrollo de ejercicios. 4o edición. Barcelona: Paidotribo.

Alter, M. J. (2008). Manual de estiramientos deportivos. 7ạ edición, 2ạ edición revisada y aumentada. Madrid: Tutor.

Arregui, J. A. y Martínez, V. (2001). Estado actual de las investigaciones sobre la flexibilidad en la adolescencia. Revista Internacional de Medicina y Ciencias de la Actividad Física y el Deporte (2) [En línea] Numero 2, Junio 2001, disponible en http://cdeporte.rediris. es/revista/revista2/artflexi.htm [Consultado el 30-072007].

Bagur, C. y Ayuso, J.M. (2001). Entrenamiento de las cualidades físicas en niños y adolescentes. Serra, J.R. (comp.), Corazón y ejercicio físico en la infancia y adolescencia. Barcelona: Masson.

Basmajian, J. V. (1982). Terapéutica por el ejercicio. 3ạ edición. Argentina: Médica panamericana.

Benavent, J. (2008). Comparación de diferentes test de campo para la evaluación de la flexibilidad general activa. Journal Fitness and Performance, Rio de Janeiro, 7, 1, 27, Ene/Feb 2008. [Consultado el 20-09-2008]. 
Bragança, M.; Bastos, A.; Salguero, A., y González, R. (2008). Flexibilidad conceptos y generalidades. Revista Educación Física y Deportes. Revista Digital - Buenos Aires - Año 12 - $\mathrm{N}^{\circ} 116$ - Enero de 2008, disponible en http: //www. efdeportes.com/efd116/flexibilidad-conceptos-y-generalidades.htm [Consultado el 13-04-2008].

Brooks, D. (2001). Libro del personal trainer. Barcelona: Paidotribo.

Cadierno, O. (2003). Clasificación y características de las capacidades motrices. Revista Educación Física y Deportes. Revista Digital - Buenos Aires - Año 9 - N 61 - Junio de 2003, disponible en http://www. efdeportes.com/efd61/capac.htm [Consultado el 13-04-2008].

Colado, J.C. (1996). Fitness en las salas de musculación. 1a edición. Barcelona: INDE.

Cortegaza, L. (2003). Capacidades y cualidades motoras. Revista Educación Física y Deportes. Revista Digital - Buenos Aires - Año 9 - $N^{\circ} 62$ - Julio de 2003, disponible en http://www. efdeportes.com/efd62/capac.htm [Consultado el 13-042008].

Cooley, B. (2007). Flexibilidad. 1aㅡ edición. Badalona: Paidotribo.

Dick, F. (1993). Principios del entrenamiento deportivo. Barcelona: Paidotribo.

Diéguez, J. (2007). Entrenamiento Funcional en Programas de Fitness. Vol. I. Barcelona: INDE.

Donskoi, D., y Zatsiorski, V. (1988). Biomecánica de los ejercicios físicos. Ciudad de La Habana: Pueblo y Educación.

Esper, P. A. (2000). El entrenamiento de la flexibilidad muscular en las divisiones formativas de baloncesto. Revista Educación Física y Deportes. Revista Digital Buenos Aires - Año 5 [En línea]. - $\mathrm{N}^{\circ} 23$ - Julio 2000, disponible en http://www.efdeportes.com/ [Consultado el 13-04-2008].

Failde, J. C. (2003). La flexibilidad. Una revisión de los conceptos más importantes a tener en cuenta para su desarrollo. Revista Comunicaciones Técnicas, № 5, 2003 pg. 9 [Consultado el 30-07-2007].

García, J. M.; Navarro, M., y Ruiz. J. A. (1996). Bases teóricas del Entrenamiento Deportivo, Principios y Aplicaciones. Madrid: Gymnos.

García, J., y Pacheco, L. (2005). Aplicación de los estiramientos en la lesión traumática. Vilar, E. y S. Sureda, (Compiladores) Fisioterapia del aparato locomotor. Madrid: MacGraw-Hill- Interamericana.

Gianikellis, K.; Vara, A.; Bote, A., y Muñoz, J. R. (2003). Análisis biomecánico para determinar la intervención muscular en los estiramientos balísticos. Revista MOTRICIDAD. European J ournal of Human Movement, 10, 85-98. [Consultado el 30-07-2007].

Gómez, P. E. (2004). Importancia del desarrollo óptimo de la flexibilidad en las Artes Marciales. Revista Educación Física y Deportes. Revista Digital - Buenos Aires Año 10 [En línea]. - $\mathrm{N}^{\circ} 69$ - Febrero de 2004, disponible en http://www.efdeportes.com/. [Consultado el 30-07-2007].

González, A. M. (2005). Algunas consideraciones acerca del entrenamiento de la flexibilidad en el taekwondo. Revista Educación Física y Deportes. Revista Digital Buenos Aires - Año 10 - $\mathrm{N}^{\circ} 87$ - Agosto de 2005, disponible en http://www. efdeportes.com/. [Consultado el 30-07-2007]. 
González, E. S.; Quesada, M., y Yanes, J. (2001). Consideraciones generales al uso de la flexibilidad en el béisbol. Revista Educación Física y Deportes. Revista Digital Buenos Aires - Año 7 [En línea]. - No 36 - Mayo de 2001, disponible en http://www.efdeportes.com/efd36/flexib.htm [Consultado el 13-04- 2008].

Harichaux, P., y Medelli, J. (2006). Tests de aptitud física y tests de esfuerzo. 1aㅡ edición. Barcelona: INDE.

Hedrick, A. (2004). Flexibilidad y entrenamiento. Flexibilidad, diferenciaciones y formas de entrenarla. Revista Alto Rendimiento: Ciencia deportiva, entrenamiento y fitness. Vol. 2, no16, ( Abril 2004). [Consultado el 13-04-2008].

Hegedüs, J. (1984). Enciclopedia de la musculación deportiva. Argentina: Stadium.

Hernández, P. E. (2007). Flexibilidad: Evidencia Científica y Metodología del Entrenamiento. PubliCE Premium. 14/03/2007. Pid: 789. [Consultado el 13-042008].

Heyward, V. H. (1996). Evaluación y Prescripción del Ejercicio. Barcelona: Paidotribo.

Kim, S. H. (2006). Flexibilidad Extrema. Guía completa de estiramientos para artes marciales. Barcelona: Paidotribo.

Manno, R. (1994). Fundamentos del entrenamiento deportivo. Barcelona: Paidotribo.

Meléndez, A. (2005). Bases fisiológicas y metodológicas del entrenamiento de la flexibilidad. Revista Comunicaciones Técnicas, № 1, 2005 pg.49.

Mora, J. (1989). Indicaciones y sugerencias para el desarrollo de la flexibilidad. 2a edición corregida y aumentada. Cádiz: Excma. Diputación Provincial de Cádiz. Servicio de Deportes.

Moras, G. (1992). Análisis critico de los actuales tests de flexibilidad. Correlación entre algunos de los tests actuales y diversas medidas antropométricas. Revista APUNTS- 1992-Vol. XXIX - 127- 137.

Pareja, L. A. (1995). La flexibilidad como capacidad fisicomotriz del hombre. Educación física y deporte, vol.17, pp. 13-30. Medellín, enero-diciembre 1995. [Consultado el 21-09-2008].

Platonov, V. (2001). Teoría General del Entrenamiento Deportivo Olímpico. Barcelona: Paidotribo

Platonov, V. N., y Bulatova, M. M. (1998). La preparación física Deporte y entrenamiento. 3o edición. Barcelona: Paidotribo.

Rivera, M. (2000). La preparación física de jóvenes futbolistas. CORPUS2000. Revista digital científico deportiva. Año 1- Edición no.1-Agosto de 2000. [Consultado el 2009-2008].

Rodríguez, P. L., y Santonja, F. (2000). Los estiramientos en la práctica físicodeportiva. Selección: Revista española e iberoamericana de medicina de la educación física y el deporte 2000; 9(4): 191-205.

Sáez, F. (2005). Una revisión de los métodos de flexibilidad y de su terminología. Revista Kronos no 7, pp. 5-16, Enero/J unio 2005. [Consultado el 13-04-2008].

Serrato, R. M. (2004). Prescripción del ejercicio. Parte III. Evaluación del Fitness. Acta Colombiana de medicina del deporte. Año 11, vol.10, no2, noviembre 2004. [Consultado el 21-09-2008].

Valbuena, R. (2007). Evaluación y normas para la clasificación de la capacidad física "flexibilidad" considerando personas entre 9 y 50 años de edad pertenecientes al Distrito Capital de la ciudad de Caracas. Revista de investigación no 61, pp. 121141, 2007. [Consultado el 20-09-2008]. 
Merino, R.; Fernández, E. (2009). Revisión sobre tipos y clasificaciones de la flexibilidad. Una nueva propuesta de clasificación. Revista Internacional de Ciencias del Deporte. 16(5), 52-70.

Vázquez, J.; Solana, R., y Pérez, B. (1997). Nuevos estiramientos fáciles. 1o edición. Madrid: Mandala Ediciones.

Vidal, J. (2004). Relationship between flexibility and sport injuries. Revista Educación Física y Deportes. Revista Digital - Buenos Aires - Año 10 [En línea]. - No 74 Julio de 2004, disponible en http://www. efdeportes.com/efd74/injur.htm [Consultado el 14-10- 2008].

Vrijens, J. (2006). Entrenamiento Razonado del Deportista. Barcelona: INDE.

Weineck, J. (1988). Entrenamiento Óptimo. Como lograr el máximo rendimiento. Barcelona: Hispano Europea. 